\title{
CCR6 blockade on regulatory $T$ cells ameliorates experimental model of multiple sclerosis
}

\author{
IZABELA JATCZAK-PAWLIK', PAWEŁ WOLINSKI', DOMINIKA KSIĄŻEK-WINIAREK', \\ MIROSŁAWA PIETRUCZUK ${ }^{3}$, ANDRZEJ GLABINSKI ${ }^{1,2}$ \\ ${ }^{1}$ Department of Propedeutics of Neurology, Medical University of Lodz, Poland \\ ${ }^{2}$ Department of Neurology and Stroke, Medical University of Lodz, Poland \\ ${ }^{3}$ Department of Laboratory Medicine, Medical University of Lodz, Poland
}

\begin{abstract}
Regulatory $T$ cells (Tregs) play a significant role in limiting damage of tissue affected by autoimmune process, which has been demonstrated in various experimental models for multiple sclerosis (MS) (mostly experimental autoimmune encephalomyelitis - EAE), rheumatoid arthritis, and type 1 diabetes. In this study, we demonstrated that Tregs increasingly migrate to central nervous system (CNS) during subsequent phases of EAE (preclinical, initial attack, and remission). In contrast, in peripheral tissues (blood, lymph nodes, and spleen), a significant accumulation of Tregs is mostly present during EAE remission. Moreover, an increased expression of CCR6 on Tregs in the CNS, blood, lymph nodes, and spleen in all phases of EAE was observed. The highest expression of CCR6 on Tregs from the CNS, lymph nodes, and spleen was noted during the initial attack of EAE, whereas in the blood, the peak expression of CCR6 was detected during the preclinical phase. The presence of Tregs in the CNS during EAE was confirmed by immunohistochemistry. To analyze additional functional significance of CCR6 expression on Tregs for EAE pathology, we modulated the clinical course of this MS model using Tregs with blocked CCR6. EAE mice, which received CCR6-deficient Tregs showed significant amelioration of disease severity. This observation suggests that CCR6 on Tregs may be a potential target for future therapeutic interventions in MS.
\end{abstract}

Key words: multiple sclerosis, Tregs, CCR6, experimental autoimmune encephalomyelitis, anti-CCR6.

(Cent Eur J Immunol 2020; 45 (3): 256-266)

\section{Introduction}

Regulatory T cells (Tregs) play a significant role in preventing development of autoimmune diseases by suppression of activation, proliferation, or differentiation of many subpopulations of immunocompetent cells, including mast cells [1], CD4+ [2] and CD8+ T cells, natural killer cells [3], and B cells [4]. However, it is strongly suggested that the major target of suppressive activity of Tregs are dendritic cells (DCs) [2], as Tregs strongly suppress DCs maturation [5]. Tregs constitute 5-15\% of peripheral CD4+ $\mathrm{T}$ cells in both mice and humans, which are identified by the expression of interleukin 2 receptor $\alpha$-chain - CD25 $[6,7]$ and the nuclear marker Foxp3, which is an essential factor for development and function of Tregs [8, 9].

Natural Tregs originate in the thymus, migrate to lymphoid organs and to the sites of inflammation, where they inhibit $\mathrm{T}$ cell proliferation and autoimmune response [10].
This migration is facilitated by the expression of several chemokine receptors [11]. The first evidence that human Tregs express chemokine receptors and respond to stimulation with chemokines was published in 2001 [12]. That study reported the expression of CCR 4 and CCR 8 on blood-borne human CD4+CD25+ Tregs and their chemotactic response to chemokines CCL22, CCL17, and CCL1 as well as to the viral chemokine vMIP-I [12]. Zou et al. reported that human Tregs accumulate in bone marrow and express CXCR4, which facilitate the migration of CD4+CD25+ Tregs to CXCL12 in bone marrow [13]. It was also shown that blocking of CXCL12/CXCR4 interaction contributes to the reduction of Tregs trafficking to the bone marrow [13]. Moreover, it was shown that 1.25-dihydroxyvitamin D3 regulates T cell differentiation and suppresses IL-17 producing T cells [14]. Other drugs, such as alpha-1 antitrypsin (AAT) [15] and chloroquine

Correspondence: Prof. Andrzej Glabinski, Department of Neurology and Stroke, Medical University of Lodz, 113 Żeromskiego St., 90-549 Lodz, Poland, e-mail: andrzej.glabinski@umed.lodz.pl Submitted: 21.11.2018; Accepted: 30.04.2019 
[16] increase CD4+FoxP3+ Treg cell population and reduce inflammation in mice, whereas $17 \beta$-estradiol can compensate for the lack of Tregs in mice with experimental autoimmune encephalomyelitis (EAE) [17]. Moreover, it was found that mesenchymal stem cells (MSCs) can suppress the proliferation, activation, and differentiation of CD4+ T cells induced to differentiate into Th1 and Th17 cells in mice with EAE [18]. This suppressive effect was associated with generation of CD4+CD25+Foxp3+ regulatory T cell subpopulation [18]. Also, it was also shown that CD4+CD25+CD69- Tregs express CCR7 and efficiently migrate to CCL19, but only a small subpopulation of CD4+CD25+CD69- Tregs express CXCR5 and migrate to CXCL13 [19]. Interestingly, upon activation, Tregs switch the chemokine receptor expression from CCR7 to CXCR5 [19]. More recently, it has been reported that two different functional subpopulations of memory Tregs can be distinguished by the expression of CCR7 [20]. Central memory Tregs $\left(\mathrm{T}_{\mathrm{CM}}\right)$ express CCR7 as opposed to effector memory Tregs $\left(\mathrm{T}_{\mathrm{EM}}\right)[20]$.

Another chemokine receptor expressed by Tregs is CCR6 [21, 22]. CCR6+ Tregs seem to represent "regulatory effector - memory" $\mathrm{T}$ cells $\left(\mathrm{T}_{\mathrm{REM}}\right)$ both in mice and humans, because they exhibit a phenotype of activation, expansion, and memory typical for effector memory T cells [21], as opposed to CCR7+ Tregs [20]. CCR6 is characteristic for the relatively new subset of $\mathrm{T}$ cells called "Th17 cells" $[22,23]$. Th17 cells are found to secret IL-17 and IL-22 [24-26], and play an important role in the induction of EAE [24, 27]. Esplugues et al. suggested that Th17 cells can acquire a regulatory phenotype with in vitro and in vivo immune-suppressive properties (rTh17) in the small intestine of mice with EAE [28].

In this study, we showed that Tregs increasingly migrate to the central nervous system (CNS) during subsequent phases of EAE (preclinical, initial attack, and remission). In peripheral tissues (blood, lymph nodes, and spleen), a significant accumulation of Tregs is present only during remission of EAE. Furthermore, we identified significant up-regulation of CCR6 expression in the CNS, blood, lymph nodes, and spleen in all phases of EAE. The expression of CCR6 on Tregs was the highest during the initial attack of EAE in the CNS and lymph nodes. To analyze further the role of CCR6 on Tregs in EAE, we attempted to modulate disease course by administration of Tregs with blocked CCR6 to EAE mice. Animals receiving these modified Tregs presented significant amelioration of the disease.

\section{Material and methods}

\section{Animals}

In all experiments, 8-12 weeks old female (SWRxSJL) F1 mice were used. All animals were purchased from the Jackson Laboratory (Bar Harbor, ME, USA) and housed at animal facility of the Medical University of Lodz, Poland under standard conditions. All experimental protocols were approved by the Animal Care Committee of the Medical University of Lodz.

\section{Experimental autoimmune encephalomyelitis induction and tissue collection}

EAE was induced by active immunization with an encephalitogenic PLP (proteolipid protein) peptide representing residues of 139-151 (PLPp: 139-151, Metabion, Martinsried, Germany) emulsified with complete Freund's adjuvant (Sigma, Poznan, Poland). Pertussis toxin (Sigma, Poznan, Poland) was injected i.v. at the day of immunization and 48 hours later, as previously described [29]. All mice were weighted and examined daily for clinical signs of EAE. The following clinical scoring scale was used: 0, no disease; 1 , decreased tail tone or slightly clumsy gait; 2 , tail atony and/or moderately clumsy gait and/or poor righting ability; 3, limb weakness; 4, limb paralysis; 5, moribund state.

For cell analysis during preclinical phase (day 10 postimmunization), the initial attack (the first three days of EAE signs), and remission of the disease, mice were anesthetized with ketamine/xylazine cocktail $(100 \mathrm{mg} / \mathrm{kg}$ of ketamine and $10 \mathrm{mg} / \mathrm{kg}$ of xylazine) (Biowet, Pulawy, Poland) administered intraperitoneally and perfused through the left cardiac ventricle using ice-cold phosphate-buffered saline (PBS, Biomed, Poland) with heparin. Preclinical phase was defined as the time with no EAE signs, initial attack of EAE as the phase when clinical signs appeared (score 1-4), and remission was identified as the time when clinical signs improved. Each investigated group contained 7 to 14 mice.

\section{Isolation of mononuclear cells from the CNS}

After being collected from an animal, the CNS (brain and spinal cord) was placed in ice-cold PBS and forced through $70 \mu \mathrm{m}$ cell strainers (BD Bioscience, Bedford, MA, USA) for a single cell suspension and then centrifuged for $10 \mathrm{~min}$ at $350 \times \mathrm{g}\left(4^{\circ} \mathrm{C}\right)$. The CNS mononuclear cells were resuspended in $40 \%$ percoll (Sigma, Poznan, Poland) and diluted with white Hank's buffered salt solution (HBSS) (Lonza, Basel, Switzerland). Then, the suspension of cells was carefully overlayered on top of the $70 \%$ percoll, diluted with red HBSS. CNS mononuclear cells were isolated by centrifugation for $40 \mathrm{~min}$ at $700 \times \mathrm{g}\left(4^{\circ} \mathrm{C}\right)$ with slow acceleration without a break. Cells were then collected from the $40 \% / 70 \%$ interface, washed and resuspended in PBS [30]. In the next step, the CNS cells were stained with trypan blue solution $0.4 \%$ (Sigma, Poznan, Poland) and counted in the Bürker chamber (Merck, Warsaw, Poland) under a light microscope objective 10x (Carl Zeiss, Axio Observer.A1, Poznan, Poland) and prepared for flow cytometry. 


\section{Isolation of mononuclear cells from blood, lymph nodes, and spleen}

After being collected from animals, the analyzed tissues (draining lymph nodes - inguinal and axillary, and spleen) were placed into ice-cold PBS and forced through $70 \mu \mathrm{m}$ cell strainers for a single cell suspension and centrifuged for $10 \mathrm{~min}$ at $350 \times \mathrm{g}\left(4^{\circ} \mathrm{C}\right)$. Blood mononuclear cells were separated on Histopaque 1077 (Sigma, Poznan, Poland) and samples were centrifuged for $25 \mathrm{~min}$ at $400 \times \mathrm{g}\left(25^{\circ} \mathrm{C}\right)$. Then, the cells were collected from the interface and washed with PBS. The remaining red cells from the blood and spleen preparation were lysed by hypotonic shock in $\mathrm{NH}_{4} \mathrm{Cl}, \mathrm{KHCO}_{3}$, and $\mathrm{Na}_{2}$ EDTA $\times 2 \mathrm{H}_{2} \mathrm{O}$ buffer, $\mathrm{pH}=7.2$ (red blood cells, RBCs buffer; Sigma, Poznan, Poland) for $5 \mathrm{~min}$ at $4^{\circ} \mathrm{C}$ [31]. Later, the cells were washed by centrifugation for $10 \mathrm{~min}$ at $400 \times \mathrm{g}\left(4^{\circ} \mathrm{C}\right)$ and resuspended in PBS. Cells isolated from the blood, lymph nodes, and spleen were stained with $0.4 \%$ trypan blue solution, and counted in Bürker chamber under a light microscope (objective 10x) and prepared for flow cytometry.

\section{Analysis of CCR6 expression on Tregs by flow cytometry}

Mononuclear cells from the CNS, blood, spleen, and lymph nodes $\left(1 \times 10^{6}\right)$ were resuspended in $100 \mu$ l of PBS and incubated with FcBlock (BD Bioscience, San Jose, CA, USA) for $15 \mathrm{~min}$ at $4^{\circ} \mathrm{C}$. After this time, specific rat antibodies for murine CD4-Pacific Blue, CD25-APC-Cy7, and CCR6-Alexa Fluor 647 (BD Bioscience, San Jose, CA, USA) were added at a predetermined optimal concentration for $15 \mathrm{~min}$ at $4^{\circ} \mathrm{C}$. The cells were washed with $1 \mathrm{ml}$ of PBS and permeabilized according to the manufacturer's protocol (eBioscience Inc., San Diego, CA, USA; mouse regulatory $\mathrm{T}$ cell staining kit, cat. no. 88-8111). FcBlock was added before incubation with Foxp3 antibody. Later, $0.5 \mu \mathrm{g}$ per test of anti-mouse/rat Foxp3-PE, Foxp3-APC, or Foxp3-PE-Cy7 antibodies (eBioscience Inc., San Diego, CA, USA) were added to each tube. The incubation lasted for $30 \mathrm{~min}$ at $4{ }^{\circ} \mathrm{C}$ in dark. Then, the cells were washed and fixed in $200 \mu \mathrm{l}$ of $1 \%$ formaldehyde/PBS solution and stored at $4^{\circ} \mathrm{C}$ until further analysis. The analysis was carried out on FACS Canto II instrument (BD Bioscience, San Jose, CA, USA) and data were analyzed using FACSDiva 6.0 software (BD Bioscience, San Jose, USA).

\section{Immunohistochemistry}

Animals were transcardially perfused with $4 \%$ formalin (Chempur, Piekary Slaskie, Poland). The CNS and spleen were removed, post-fixed for 2 days in $4 \%$ formalin solution, and embedded in paraffin. Sections (10 $\mu \mathrm{m}$ thick) were placed onto glass slides (Menzel-Glaser, Braunschweig, Germany). Slides with paraffin-embedded sections were deparaffinized by immersion in xylene (Sigma, Poznan, Poland), followed by rehydration in ethanol
(POCH, Gliwice, Poland). Antigen retrieval was done with $10 \mathrm{mM}$ citrate buffer, pH 6.0 (Sigma, Poznan, Poland). Tissue sections were incubated overnight at $4{ }^{\circ} \mathrm{C}$, with primary rat anti-Foxp3 antibody (eBioscience Inc., San Diego, CA, USA) at 1:500 dilution. Secondary biotinylated rabbit anti-rat antibodies were purchased from DAKO North America Inc. (Carpinteria CA, USA) and ABC kit from Vector Laboratories (Peterborough, United Kingdom). Staining was performed using diaminobenzidine substrate (Sigma, Poznan, Poland) and counterstaining was done with hematoxylin-eosin (Sigma, Poznan, Poland).

\section{Modulation of EAE using CCR6-deficient Tregs}

The cells isolated from drained lymph nodes (inguinal and axillary) of animals with EAE remission ( $3^{\text {rd }}$ and $4^{\text {th }}$ day) were counted in Bürker chamber under a light microscope objective 10x. Next, Tregs were sorted using CD4+CD25+ regulatory $\mathrm{T}$ cell isolation kit, according to the manufacturer's instruction. Approximately, $4 \mathrm{mln}$ of Tregs were obtained from each mouse. Half of these cells were incubated with anti-CCR6 antibody (R\&D Systems, Minneapolis, MN, USA) and the other half was left unchanged. Concentration of anti-CCR6 antibody was $4.5 \mu \mathrm{g} / \mathrm{ml}$ per $1 \mathrm{mln}$ of Tregs resuspended in $100 \mu \mathrm{l}$ of PBS. Tregs were incubated with antibody for $20 \mathrm{~min}$ at $4^{\circ} \mathrm{C}$. After this time, $1 \mathrm{ml}$ of PBS was added to the cells and unbound antibody was removed by centrifugation $(10 \mathrm{~min}, 350 \times \mathrm{g}$, $4^{\circ} \mathrm{C}$ ). CCR6 protein expression on Tregs and on Tregs with blocked CCR6 was analyzed by flow cytometry.

Tregs ( $2 \mathrm{mLn}$ cells) and Tregs with blocked CCR6 ( 2 mln cells) were separately applied to two different groups of immunized mice during preclinical phase of EAE ( $7^{\text {th }}$ or $8^{\text {th }}$ day post-immunization). Both groups consisted of 6 mice. Tregs were given slowly, intravenously to the mouse tail vein at a concentration of $1 \mathrm{mln} / \mathrm{ml}$. The third group of animals were the control group and consisted of 5 mice with unmanipulated EAE to show natural course of the disease. All animals were examined daily for 39 days, and EAE scores were provided by two independent observers.

\section{Statistical analysis}

For statistical analysis, non-parametric Kruskal-Wallis and Mann-Whitney tests with Bonferroni correction were used. A value of $p<0.05$ was considered statistically significant.

\section{Results}

\section{Accumulation of Tregs in the CNS and peripheral tissues during EAE}

The number of Tregs (CD4+CD25+Foxp3+) isolated from the CNS increased significantly during all three analyzed phases of EAE (preclinical, initial attack, and 
remission) in comparison to control unimmunized group $(p=0.03, p=0.0001$, and $p=0.002$, respectively; Mann-Whitney test) (Figs. 1 and 2A). During remission of EAE, the number of Tregs accumulating in the CNS was significantly higher than in the preclinical phase and initial attack ( $p=0.03$ and $p=0.0003$, respectively; Mann-Whitney test) (Fig. 2A).

The number of Tregs circulating in the blood of control mice, animals from preclinical phase, initial attack, and remission of EAE was significantly different ( $p=0.0001$, Kruskal-Wallis test). Further analysis revealed that the number of Tregs was significantly higher during EAE remission than in the blood of control mice, preclinical phase, and initial attack of $\operatorname{EAE~}(p=0.0009, p=0.03$, and $p=0.012$, respectively; Mann-Whitney test) (Fig. 2B).

The number of Tregs isolated from the lymph nodes was also different in groups of control mice, animals from preclinical phase, initial attack, and remission of EAE (Kruskal-Wallis test, $p=0.0002$ ). During the remission of EAE, the number of Tregs in lymph nodes was significantly higher than in the control group and the group of initial EAE attack ( $p=0.004$ and $p=0.04$, respectively; Mann-Whitney test) (Fig. 2C).

Similarly, there was also a significant difference in the number of Tregs detected in the spleen between the control mice, mice from preclinical phase, first attack, and remission of EAE ( $p=0.0002$, Kruskal-Wallis test). The highest number of Tregs was observed during the remission of EAE. Significant differences in the number of Tregs were observed between the following groups of mice: remission vs. control group, remission vs. preclinical phase, and preclinical vs. control mice ( $p=0.03, p=0.04$, and $p=0.04$, respectively; Mann-Whitney test) (Fig. 2D).

\section{Immunohistochemical localization of Tregs in the CNS during EAE}

Foxp3-positive cells were detected in the CNS during different stages of EAE with immunohistochemistry. Their morphology suggested that they were lymphocytes. A minimal number of Tregs was also detected in the CNS of control unimmunized mice (Fig. 3A). Their number increased evidently during the initial attack (Fig. 3B) as well as in remission of EAE (Fig. 3C). Positive control for staining procedure were Tregs detected in spleen (Fig. 3D).

\section{Expression of CCR6 on Tregs from the CNS and peripheral tissues during EAE}

Flow cytometry analysis of CCR6 expression on Tregs from the CNS of control mice, animals from preclinical phase, initial attack, and remission of EAE showed significant differences ( $p=0.0005$, Kruskal-Wallis test). Further analysis confirmed an increase of this expression on Tregs from the CNS during preclinical phase, initial attack, and
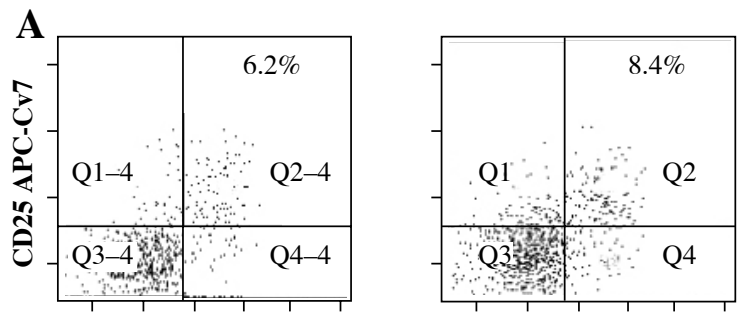

Foxp3 PE-Cy7

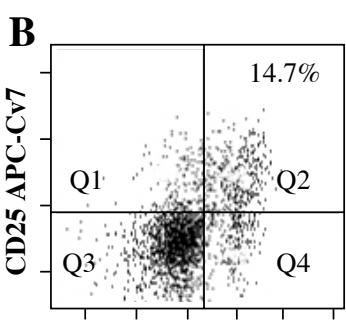

Foxp3 PE-Cy7

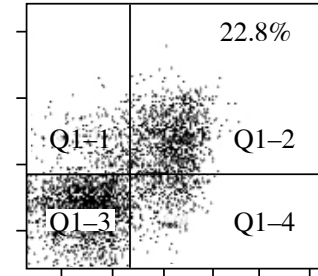

Foxp3 PE
Fig. 1. Flow cytometry analysis of Tregs (CD4+CD25+Foxp3+ T cells) from the central nervous system (CNS) of control normal animals (A) and from the CNS of experimental autoimmune encephalomyelitis (EAE) animals obtained during three successive phases of the disease: preclinical (B), initial attack (C), and remission (D). Dot plots are shown for the populations gated on CD4+ T cells and display surface staining of CD25 versus Foxp3. Data is representative of individually analyzed mice $(n=14$ per group). Numbers represent the percentage of CD25+Foxp3+ cells. Cells from the CNS of control unimmunized mice were pooled from 4 mice

remission of the disease compared to controls ( $p=0.037$, $p=0.037$, and $p=0.023$, respectively; Mann-Whitney test) (Fig. 4A). Also, CCR6 expression on Tregs was significantly higher in the initial attack rather than in preclinical phase and remission of EAE ( $p=0.047$ and $p=0.037$, respectively; Mann-Whitney test) (Fig. 4A).

CCR6 protein expression on blood Tregs from control mice, animals from preclinical phase, initial attack, and remission of EAE was significantly different $(p>0.05$, Kruskal-Wallis test). The expression of CCR6 on Tregs from the blood during preclinical phase, initial attack, and remission of EAE was significantly higher than in control unimmunized group $(p=0.0035, p=0.0035$, and $p=0.011$, respectively; Mann-Whitney test) (Fig. 4B).

Additionally, the expression of CCR6 was analyzed on Tregs isolated from the lymph nodes of control unimmunized mice and animals from preclinical phase, initial attack, and remission of EAE. This analysis revealed significant difference of CCR6 expression on Tregs ( $p=0.0003$, Kruskal-Wallis test). CCR6 expression on Tregs from the preclinical phase and initial attack of EAE was higher than in the control group $(p=0.0005$ and $p=0.01$, respectively, Mann-Whitney test). Moreover, the expression of this 

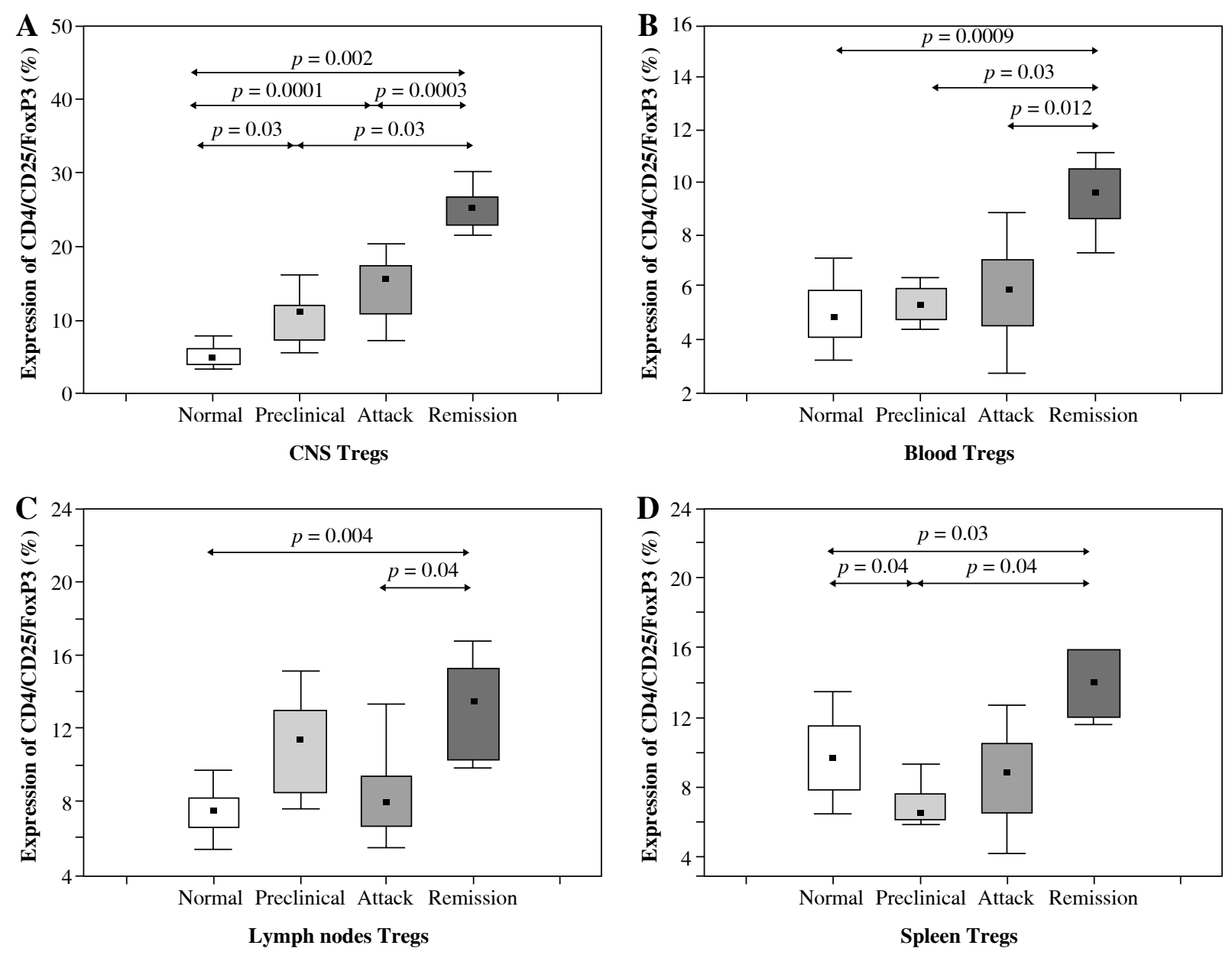

Fig. 2. Accumulation of Tregs during three consecutive phases of experimental autoimmune encephalomyelitis (EAE) (preclinical, initial attack, and remission) in the central nervous system (CNS) (A), blood (B), lymph nodes (C), and spleen (D) analyzed by flow cytometry. Cells from the CNS of control unimmunized mice were pooled from 6 mice. Data is derived from a CD4+ cell gate, $n=14$ per group, Mann-Whitney test with Bonferroni correction
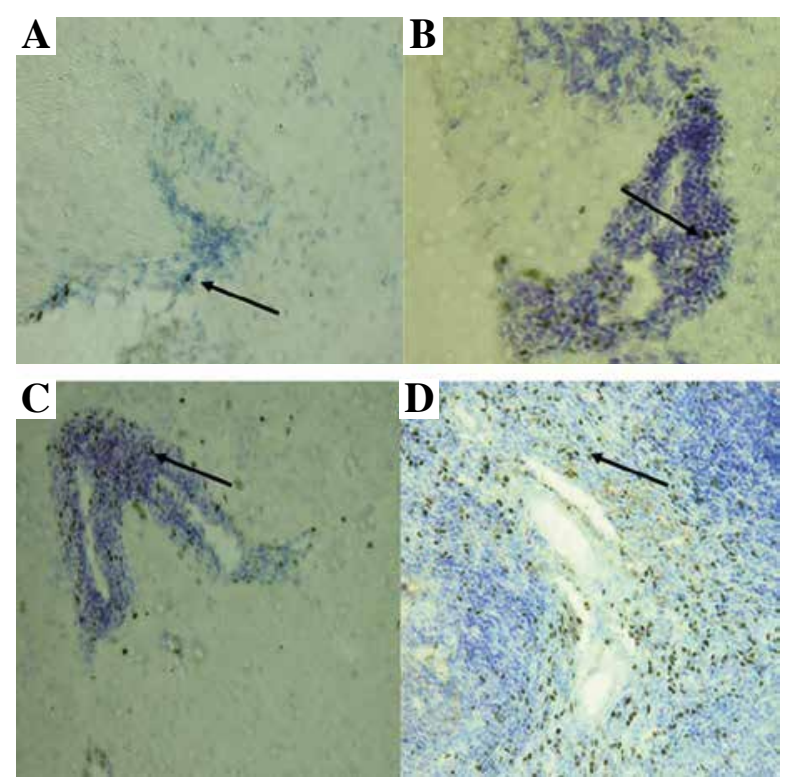

Fig. 3. Localization of Tregs (Foxp3-positive cells) (brown cells, arrows) in the brain and spleen of experimental autoimmune encephalomyelitis (EAE) mice. Staining was developed using diaminobenzidine substrate and sections were counterstained with hematoxylin and eosin. Representative brain sections are shown from: healthy control (A), initial attack (B), and remission (C) of the disease; section from the spleen (D) served as a positive control for staining (original magnification $20 \times$ ) 

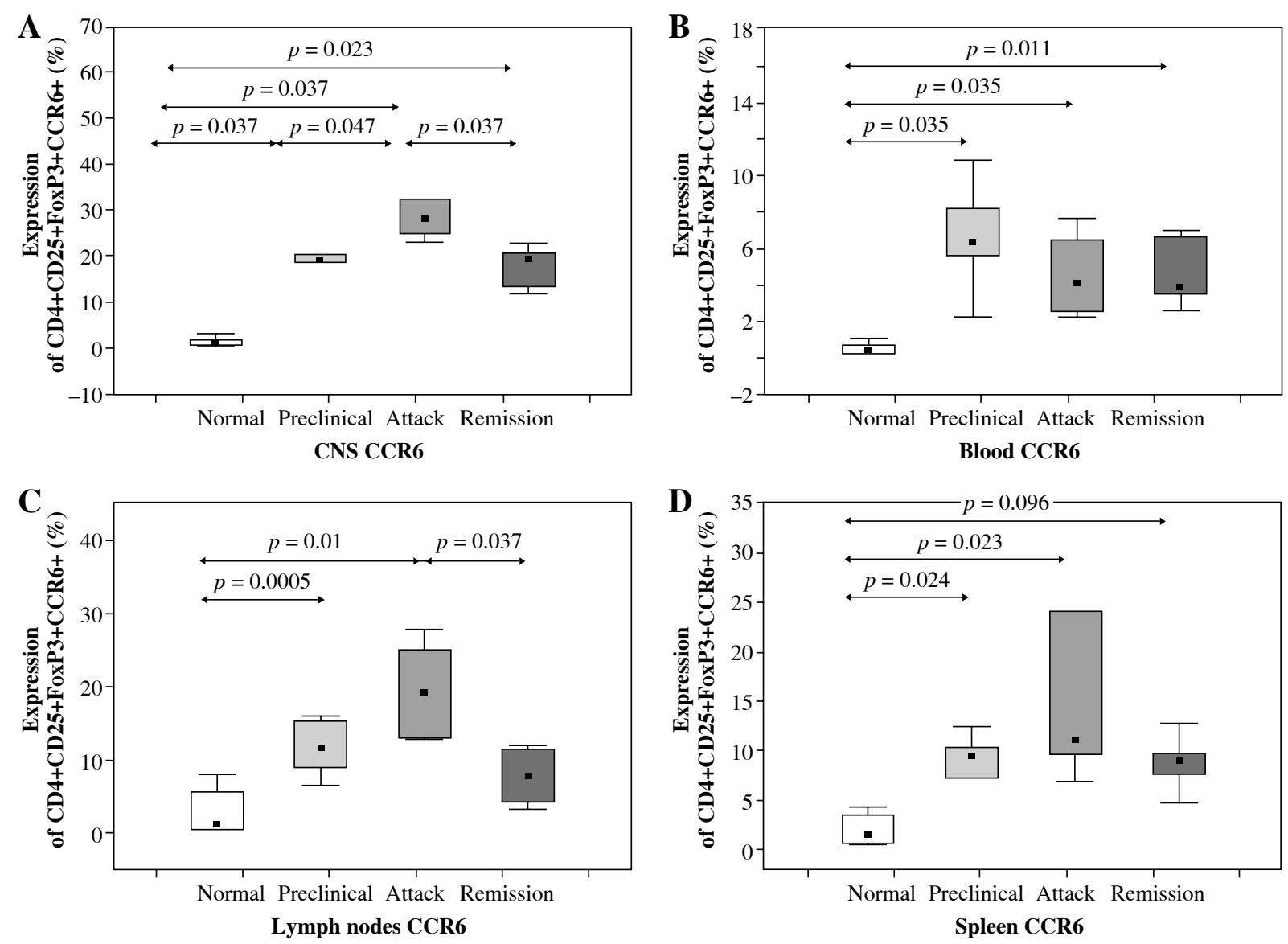

Fig. 4. CCR6 protein expression on Tregs from the central nervous system (CNS) (A), blood (B), lymph nodes (C), and spleen (D) of animals from preclinical phase, initial attack, and remission of experimental autoimmune encephalomyelitis (EAE) assessed by flow cytometry. Data is derived from a CD4+ Foxp3+ cell gate, $n=7$ per group, Mann-Whitney test with Bonferroni correction

receptor was lower during remission in comparison to the initial attack ( $p=0.037$, Mann-Whitney test) (Fig. 4C).

CCR6 protein expression on Tregs from the spleen of control mice, animals from preclinical phase, initial attack, and remission of EAE was significantly different ( $p=0.0001$, Kruskal-Wallis test). CCR6 expression on Tregs from the spleen increased during the preclinical phase, initial attack, and the remission of EAE compared to the control group ( $p=0.024, p=0.0023$, and $p=0.0096$, respectively; Mann-Whitney test) (Fig. 4D).

\section{Modulation of EAE by delivery of CCR6-deficient Tregs}

To analyze the functional effect of CCR6 deficiency on Tregs on the clinical course of EAE, Tregs with blocked CCR6 were delivered to recipient EAE mice (as described in "Material and methods" section). CCR6 protein expression on unmanipulated Tregs from lymph nodes was at the level of $17.2 \%$ (Fig. 5A) and on Tregs blocked with anti-CCR6 antibody was $5.2 \%$, as measured by flow cytometry (Fig. 5B).

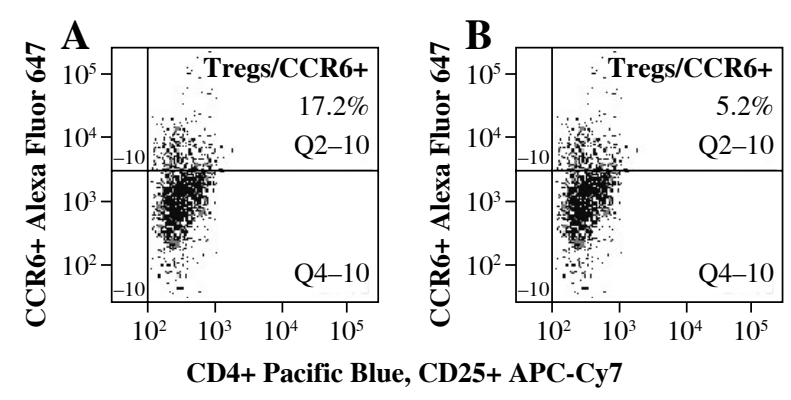

Fig. 5. Expression of CCR6 on Tregs from lymph nodes of experimental autoimmune encephalomyelitis (EAE) mice with disease remission before (A) and after (B) blockade of CCR6 with specific antibody (concentration $4.5 \mu \mathrm{g} / \mathrm{ml}$ ) assessed by flow cytometry

The onset of EAE was seen in all three analyzed groups at the same time, but the effector phase of disease significantly changed in the group with blocked CCR6 Tregs (Fig. 6). These animals showed significantly milder disease. Statistically significant amelioration of severity of the disease 


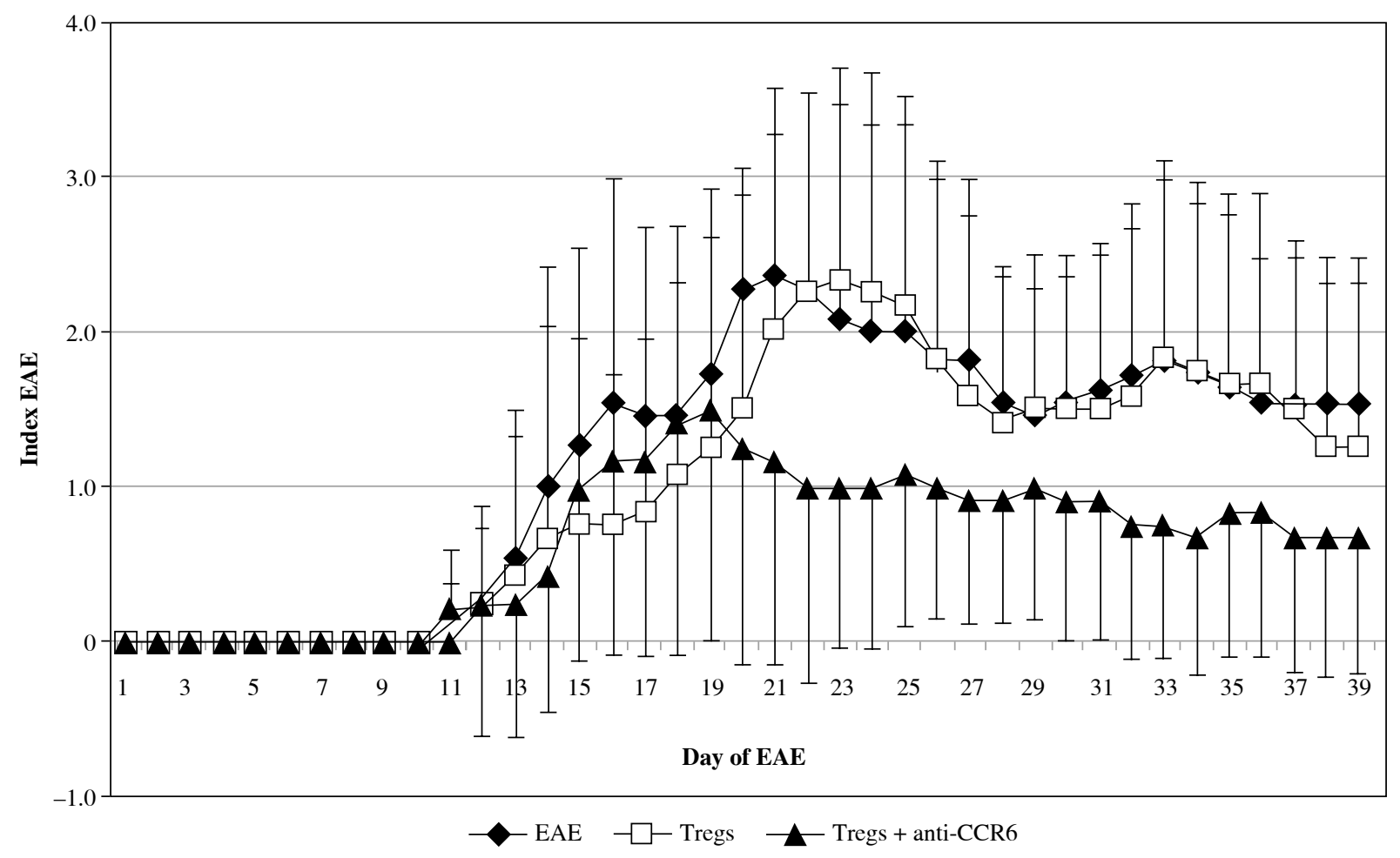

Fig. 6. Modulation of experimental autoimmune encephalomyelitis (EAE) clinical course by passive transfer of Tregs or Tregs with blocked CCR6. Tregs were isolated and blocked as described in "Material and methods" section. Data is expressed as the mean index of EAE for all mice in each group. Bars shows SD (EAE - unmanipulated EAE group used as morbidity control, Tregs - EAE group which received unblocked Tregs, Tregs + anti-CCR6 - EAE group which received Tregs with blocked CCR6)

was seen at days 20-23 (EAE vs. CCR6-deficient Tregs: $p=0.049, p=0.035, p=0.023$, and $p=0.044$, for consecutive days, respectively; Mann-Whitney test). During the remission phase, unmanipulated EAE have shown higher index of EAE than animals with CCR6-deficient Tregs ( $p=0.035$ for day 27, and $p=0.044$ for day 28; MannWhitney test). When the second attack of the disease appeared in untreated animals, the group of EAE mice, which received Tregs with blocked CCR6, became again significantly less affected ( $p=0.019$ for day 32, $p=0.018$ for day 33, and $p=0.013$ for day 34; Mann-Whitney test). The same situation was observed in the second remission ( $p=0.02$ for days 37-39, Mann-Whitney test) (Fig. 6).

The first and second attack of EAE was almost invisible in mice that received Tregs with blocked CCR6 in comparison to mice with unmanipulated Tregs. Statistical differences in disease severity between these groups appeared at the first attack of disease (days 22-25, $p=0.023$, $p=0.016, p=0.025$, and $p=0.04$, respectively for consecutive days; Mann-Whitney test) and at the second attack of EAE (days 32-34, $p=0.047, p=0.036$, and $p=0.026$, respectively; Mann-Whitney test). During the second remission of disease in contrast to the first one, mice with
Tregs without CCR6 were again significantly less affected ( $p=0.043$ for day 37, Mann-Whitney test) (Fig. 6).

\section{Discussion}

Tregs are induced in periphery after targeting foreign antigens presented by dendritic cells (DCs) [32]. To travel to various tissue sites during development and inflammatory conditions, they need to regulate the expression of receptors for adhesion of molecules and chemokines [11, 33, 34]. Tregs in mice and humans express many trafficking chemokine receptors, including CCR4 [12, 35, 36], CCR5 [37], CCR7 [38], CCR8 [12], and CCR10 [39]. They can be upregulated in inflamed tissues [40-44] as well as in secondary lymphoid organs $[45,46]$.

In the present study, we have reported an increased accumulation of Tregs in the CNS during preclinical phase, initial attack, and remission of EAE, an experimental model of multiple sclerosis (MS). Immunohistochemical staining confirmed the increased number of Tregs during EAE in the CNS. Additionally, we demonstrated that the number of Tregs in the blood, lymph nodes, and spleen was the highest during the remission of EAE. Similar results for the CNS and lymph nodes were presented by Villares et al. [47]. 
There are several studies emphasizing the important role of Tregs in suppression of autoimmune diseases [48, 49]. It has been shown that amelioration of EAE is possible by transferring of Tregs from nadve mice [40, 50]. Moreover, a depletion of CD25+ T cells inhibited the recovery from EAE $[43,50,51]$. We observed that Tregs from lymph nodes of mice from EAE remission phase and transferred into recipient mice during preclinical phase of EAE did not significantly changed the course and severity of the disease. Opposite results were obtained by McGeachy et al., but they were using a different experimental protocol. In their study, lower number of unmanipulated Tregs $\left(2 \times 10^{4}\right.$ cells $)$ was transferred much earlier, either 1 day before or 1 day after immunization of mice [43]. These authors showed that CNS-derived Tregs significantly reduced signs of EAE [43].

Here, we observed increased expression of CCR6 on Tregs during three successive phases of EAE (preclinical, initial attack, and remission). Besides elevated expression of CCR6 on Tregs from the CNS, such expression increased also on Tregs from the blood, lymph nodes, and spleen. Interestingly, the pattern of CCR6 expression on Tregs was similar in all three analyzed phases of EAE in the blood and spleen in contrast to the expression observed in the CNS and lymph nodes. In the CNS and lymph nodes, we found significantly increased expression of CCR6 on Tregs during the initial attack when compared to the remission of EAE. Kleinewietfeld et al. reported similar results for CNS [21]. Additionally, during the remission of EAE, we observed an increased number of Tregs in lymph nodes, but subpopulation of CCR6+ Tregs decreased. Probably, during remission, CCR6+ Tregs leave the lymph nodes or they downregulate CCR6 at that time.

Similarly, the expression of CCL20, a CCR6 ligand, in the CNS and lymph nodes differs during all three phases of EAE [52]. This expression increases during the preclinical phase of EAE, which later is strongly upregulated during the initial attack and returns to the baseline level during the remission of EAE [5, 52]. The highest production of CCL20 in lymph nodes of EAE mice was observed during preclinical phase of EAE [52]. Probably, CCR6+ Tregs accumulate in the CNS and lymph nodes during the preclinical phase and/or attack of EAE. Whilst the disease decreases, the level of CCL20 both in the CNS and lymph nodes diminish, which correlates with decreased number of CCR6+ Tregs in tissues. This correlation suggests that CCL20 may stimulate the migration of CCR6+ Tregs during EAE.

CCR6 plays a role in regulating the migration of $\mathrm{T}$ cells and B cells patrolling the normal CNS [23]. Similarly, CCR6+ T cells freshly isolated from blood of normal donors can migrate to CCL20 in vitro [53]. This observation confirms that CCR6 is essential not only in pathological but also in physiological processes of the CNS. Furthermore, the expression of CCR6 is detected on Tregs and CD45RO+ effector cells [23, 54]. It is possible that in a steady state condition (healthy organism), the major activity of Tregs is to suppress DCs, mostly their maturation [5]. Antigen-activated CD45RO+ cells are able to fully release immature DCs from suppression, which activates DCs and abrogates Tregs ability to downregulate DCs markers [5], including co-stimulatory molecules CD80/CD86 [2, 55], CCR7, and HLA-DR [54] as well as proinflammatory cytokines [55]. It is known that highly activated DCs can be resistant to suppression $[5,56]$. It was reported that the interaction of CD40 on DCs with CD154 (CD40L) on CD4+CD25- T cells releases DCs from control of Tregs. This has been confirmed by blocking of CD40 on activated DCs in vitro [5].

CCL20 - the only known ligand for CCR6 $[57,58]$ is required for the recruitment of $\mathrm{CD} 45 \mathrm{RO}+$ memory $\mathrm{T}$ cells to the site of antigen presentation, which leads to immune cell activation $[23,53]$. It was suggested that CD45RO+ cells expressing CCR6 play an important role in the early phase of inflammatory response [53]. Both CD4+ T cells [57] and immature dendritic cells [59] express CCR6 and traffic to the lymph nodes towards CCL20, which is expressed by endothelial cells upon activation by TNF alpha [60]. TNF alpha is important for innate immunity and is expressed in lymph nodes $[60,61]$. Reboldi et al. have shown that CCL20 can be constitutively expressed by endothelial cells from the choroid plexus in CNS ventricles [23].

Recently, it was reported that immunocompetent cells that enter the CNS very early during inflammatory response (the first wave) are autoreactive Th17 cells expressing CCR6, which lead to a second wave of migration of antigen non-specific T cells to the CNS [23]. The first influx of CCR6+ Th17 cells is needed for cellular activation within inflammatory foci, and leads to a production of inflammatory cytokines and chemokines, which in turn activate the blood-brain barrier (BBB) and initiate the influx of large number of inflammatory effector cells [23]. It was also confirmed that Th17 cells, but not Th1 and Th2 T cells, highly express CCR6 [22]. CCR6 may be essential in the priming phase of EAE and is not involved in the effector phase of EAE [62]. It has been reported that administration of anti-CCR6 [62] and anti-CCL20 [52] antibodies before passive transfer of PLP 139-151 reactive lymphocytes had not changed the course of EAE. Therefore, it was suggested that CCR6 is not involved in the effector phase of EAE [62]. Additionally, the lack of CCR6 on Th17 cells impaired the recruitment of those cells to the CNS during EAE [22]. Moreover, neutralization of IL-17 strongly inhibits EAE [24, 27]. Those observations confirmed the essential role of Th17 cells in the induction of EAE and the important function of CCR6 in migration of those cells to inflamed tissues.

In this study, we investigated whether CCR6 on Tregs from EAE animals given to recipients has an impact on 
disease's course and severity. We showed that Tregs with blocked CCR6 may decrease acute EAE severity in recipients. This group of mice developed significantly milder neurological damage during the first and the second attack of disease compared to the control EAE group. It seems that CCR6 on Tregs is needed for their migration to the CNS, and its absence significantly reduce the signs of EAE.

Villares et al. showed that CCR6+/+ Tregs transferred to CCR6-/- mice before EAE induction reduced the severity of EAE. They concluded that CCR6 is required for Tregs to downregulate EAE [47]. However, the results of this study are very difficult to compare with ours because of different experimental protocols. Firstly, they transferred CCR6+/+ Tregs much earlier, even before EAE induction. We delivered Tregs with blocked CCR6 to recipients on days 7-8 after EAE induction during preclinical phase of the disease. Secondly, we isolated Tregs from EAE mice during remission, whereas Villares et al. used normal (unimmunized) animals [47]. Moreover, the recipient animals in their study were CCR6-/- animals, whereas we used wild type mice. Our EAE models were also different. They used MOG-induced model in C57B1/6 mice, while our model was PLP-induced in (SWRxSJL) F1 mice. Additionally, our Tregs were collected from draining lymph nodes, whereas Villares et al. collected Tregs from spleen [47]. All above-mentioned differences in study design may justify different conclusions from our experiments. However, the results from both studies taken together may indicate the important, but complex role of CCR6 on Tregs in EAE pathogenesis. Further studies are needed to clarify these observations.

In our study, we demonstrated that Tregs increasingly migrate to the CNS during subsequent phases of EAE (preclinical, initial attack, and remission). Moreover, we observed an increased expression of CCR6 on Tregs in the CNS, blood, lymph nodes, and spleen in all phases of EAE. To further analyze the functional significance of such expression in EAE pathology, we modulated the clinical course of this MS model using Tregs with blocked CCR6. EAE mice, which received CCR6-deficient Tregs, showed significant amelioration of the disease severity. This observation suggests that CCR6 on Tregs may be a potential target for the future therapeutic interventions in MS.

\section{Acknowledgements}

This work was supported by research grant Innochem LSHB-CT-2005-518161 from 6FPEU, grant 263/6.PR UE/2006/7 from the Polish Ministry of Science and Higher Education, and by the Medical University of Lodz, 503/5062-01/503-51-002.

The authors declare no conflict of interest.

\section{References}

1. Lu LF, Lind EF, Gondek DC, et al. (2006): Mast cells are essential intermediaries in regulatory T-cell tolerance. Nature 442: 997-1002.

2. Tran DQ, Shevach EM (2009): Therapeutic potential of FOXP3(+) regulatory $T$ cells and their interactions with dendritic cells. Hum Immunol 70: 294-299.

3. Giroux M, Yurchenko E, St-Pierre J, et al. (2007): T regulatory cells control numbers of NK cells and CD8alpha+ immature dendritic cells in the lymph node paracortex. J Immunol 179: 4492-4502.

4. Zhao DM, Thornton AM, DiPaolo RJ, Shevach EM (2006): Activated CD4+CD25+ T cells selectively kill B lymphocytes. Blood 107: 3925-3932.

5. Serra P, Amrani A, Yamanouchi J, et al. (2003): CD40 ligation releases immature dendritic cells from the control of regulatory CD4+CD25+ T cells. Immunity 19: 877-889.

6. Holm TL, Nielsen J, Claesson MH (2004): CD4+CD25+ regulatory T cells: I. Phenotype and physiology. Apmis 112: 629-641.

7. Baecher-Allan C, Viglietta V, Hafler DA (2004): Human CD4+CD25+ regulatory T cells. Semin Immunol 16: 89-98.

8. Ono M, Yaguchi H, Ohkura N, et al. (2007): Foxp3 controls regulatory T-cell function by interacting with AML1/Runx1. Nature 446: 685-689.

9. Zheng Y, Rudensky AY (2007): Foxp3 in control of the regulatory T cell lineage. Nat Immunol 8: 457-462.

10. Wing K, Fehervari Z, Sakaguchi S (2006): Emerging possibilities in the development and function of regulatory T cells. Int Immunol 18: 991-1000.

11. Sonar SA, Lal G (2017): Differentiation and Transmigration of CD4 T Cells in Neuroinflammation and Autoimmunity. Front Immunol 8: 1695.

12. Iellem A, Mariani M, Lang R, et al. (2001): Unique chemotactic response profile and specific expression of chemokine receptors CCR4 and CCR8 by $\mathrm{CD} 4(+) \mathrm{CD} 25(+)$ regulatory T cells. J Exp Med 194: 847-853.

13. Zou L, Barnett B, Safah H, et al. (2004): Bone marrow is a reservoir for $\mathrm{CD} 4+\mathrm{CD} 25+$ regulatory $\mathrm{T}$ cells that traffic through CXCL12/CXCR4 signals. Cancer Res 64: 84518455 .

14. Chang JH, Cha HR, Lee DS, et al. (2010): 1,25-Dihydroxyvitamin D3 inhibits the differentiation and migration of $\mathrm{T}(\mathrm{H}) 17$ cells to protect against experimental autoimmune encephalomyelitis. PloS One 5: e12925.

15. Subramanian S, Shahaf G, Ozeri E, et al. (2011): Sustained expression of circulating human alpha-1 antitrypsin reduces inflammation, increases CD4+FoxP3+ Treg cell population and prevents signs of experimental autoimmune encephalomyelitis in mice. Metab Brain Dis 26: 107-113.

16. Thome R, Moraes AS, Bombeiro AL, et al. (2013): Chloroquine treatment enhances regulatory $\mathrm{T}$ cells and reduces the severity of experimental autoimmune encephalomyelitis. PLoS One 8: e65913.

17. Subramanian S, Yates M, Vandenbark AA, Offner H (2011): Oestrogen-mediated protection of experimental autoimmune encephalomyelitis in the absence of Foxp3+ regulatory T cells implicates compensatory pathways including regulatory B cells. Immunology 132: 340-347.

18. Luz-Crawford P, Kurte M, Bravo-Alegria J, et al. (2013): Mesenchymal stem cells generate a CD4+CD25+Foxp3+ regulatory $\mathrm{T}$ cell population during the differentiation process of Th1 and Th17 cells. Stem Cell Res Ther 4: 65. 
19. Lim HW, Hillsamer P, Kim CH (2004): Regulatory T cells can migrate to follicles upon $\mathrm{T}$ cell activation and suppress GC-Th cells and GC-Th cell-driven B cell responses. J Clin Invest 114: 1640-1649.

20. Smigiel KS, Richards E, Srivastava S, et al. (2014): CCR7 provides localized access to IL-2 and defines homeostatically distinct regulatory T cell subsets. J Exp Med 211: 121-136.

21. Kleinewietfeld M, Puentes F, Borsellino G, et al. (2005): CCR6 expression defines regulatory effector/memory-like cells within the CD25(+)CD4+ T-cell subset. Blood 105: 2877-2886.

22. Yamazaki T, Yang XO, Chung Y, et al. (2008): CCR6 regulates the migration of inflammatory and regulatory $\mathrm{T}$ cells. J Immunol 181: 8391-8401.

23. Reboldi A, Coisne C, Baumjohann D, et al. (2009): C-C chemokine receptor 6-regulated entry of TH-17 cells into the CNS through the choroid plexus is required for the initiation of EAE. Nat Immunol 10: 514-523.

24. Langrish CL, Chen Y, Blumenschein WM, et al. (2005): IL-23 drives a pathogenic T cell population that induces autoimmune inflammation. J Exp Med 201: 233-240.

25. Chung Y, Yang X, Chang SH, et al. (2006): Expression and regulation of IL-22 in the IL-17-producing CD4+ T lymphocytes. Cell Res 16: 902-907.

26. Liang SC, Tan XY, Luxenberg DP, et al. (2006): Interleukin (IL)-22 and IL-17 are coexpressed by Th17 cells and cooperatively enhance expression of antimicrobial peptides. J Exp Med 203: 2271-2279.

27. Park H, Li Z, Yang XO, et al. (2005): A distinct lineage of $\mathrm{CD} 4 \mathrm{~T}$ cells regulates tissue inflammation by producing interleukin 17. Nat Immunol 6: 1133-1141.

28. Esplugues E, Huber S, Gagliani N, et al. (2011): Control of TH17 cells occurs in the small intestine. Nature 475: 514-518.

29. Glabinski AR, Tani M, Tuohy VK, Ransohoff RM (1997): Murine experimental autoimmune encephalomyelitis: a model of immune-mediated inflammation and multiple sclerosis. Methods Enzymol 288: 182-190.

30. Wojkowska DW, Szpakowski P, Ksiazek-Winiarek D, et al. (2014): Interactions between neutrophils, Th17 cells, and chemokines during the initiation of experimental model of multiple sclerosis. Mediators Inflamm 2014: 590409.

31. Bielecki B, Jatczak-Pawlik I, Wolinski P, et al. (2015): Central Nervous System and Peripheral Expression of CCL19, CCL21 and Their Receptor CCR7 in Experimental Model of Multiple Sclerosis. Arch Immunol Ther Exp 63: 367-376.

32. Kretschmer K, Apostolou I, Hawiger D, et al. (2005): Inducing and expanding regulatory $\mathrm{T}$ cell populations by foreign antigen. Nat Immunol 6: 1219-1227.

33. Butcher EC, Williams M, Youngman K, et al. (1999): Lymphocyte trafficking and regional immunity. Adv Immunol 72: 209-253.

34. Cyster JG (2000): Leukocyte migration: scent of the T zone. Curr Biol 10: R30-33.

35. Lee I, Wang L, Wells AD, et al. (2005): Recruitment of Foxp3+ T regulatory cells mediating allograft tolerance depends on the CCR4 chemokine receptor. J Exp Med 201: 1037-1044.

36. Ishida T, Ishii T, Inagaki A, et al. (2006): Specific recruitment of CC chemokine receptor 4-positive regulatory $\mathrm{T}$ cells in Hodgkin lymphoma fosters immune privilege. Cancer Res 66: 5716-5722.

37. Wysocki CA, Burkett SB, Panoskaltsis-Mortari A, et al. (2004): Differential roles for CCR5 expression on donor
T cells during graft-versus-host disease based on pretransplant conditioning. J Immunol 173: 845-854.

38. Szanya V, Ermann J, Taylor C, et al. (2002): The subpopulation of $\mathrm{CD} 4+\mathrm{CD} 25+$ splenocytes that delays adoptive transfer of diabetes expresses L-selectin and high levels of CCR7. J Immunol 169: 2461-2465.

39. Eksteen B, Miles A, Curbishley SM, et al. (2006): Epithelial inflammation is associated with CCL28 production and the recruitment of regulatory T cells expressing CCR10. J Immunol 177: 593-603.

40. Kohm AP, Carpentier PA, Anger HA, Miller SD (2002): Cutting edge: CD4+CD25+ regulatory T cells suppress antigen-specific autoreactive immune responses and central nervous system inflammation during active experimental autoimmune encephalomyelitis. J Immunol 169: 4712-4716.

41. Mottet C, Uhlig HH, Powrie F (2003): Cutting edge: cure of colitis by CD4+CD25+ regulatory T cells. J Immunol 170: 3939-3943.

42. Lundsgaard D, Holm TL, Hornum L, Markholst H (2005): In vivo control of diabetogenic T-cells by regulatory $\mathrm{CD} 4+\mathrm{CD} 25+$ T-cells expressing Foxp3. Diabetes 54: 1040-1047.

43. McGeachy MJ, Stephens LA, Anderton SM (2005): Natural recovery and protection from autoimmune encephalomyelitis: contribution of CD4+CD25+ regulatory cells within the central nervous system. J Immunol 175: 3025-3032.

44. Ott PA, Anderson MR, Tary-Lehmann M, Lehmann PV (2005): CD4+CD25+ regulatory T cells control the progression from periinsulitis to destructive insulitis in murine autoimmune diabetes. Cell Immunol 235: 1-11.

45. Sarween N, Chodos A, Raykundalia C, et al. (2004): CD4+CD25+ cells controlling a pathogenic CD4 response inhibit cytokine differentiation, CXCR-3 expression, and tissue invasion. J Immunol 73: 2942-2951.

46. Viglietta V, Baecher-Allan C, Weiner HL, Hafler DA (2004): Loss of functional suppression by CD4+CD25+ regulatory T cells in patients with multiple sclerosis. J Exp Med 199: 971-979.

47. Villares R, Cadenas V, Lozano M, et al. (2009): CCR6 regulates EAE pathogenesis by controlling regulatory CD4+ T-cell recruitment to target tissues. Eur J Immunol 39: 16711681.

48. Danikowski KM, Jayaraman S, Prabhakar BS (2017): Regulatory $\mathrm{T}$ cells in multiple sclerosis and myasthenia gravis. J Neuroinflammation 14: 117.

49. Knochelmann HM, Dwyer CJ, Bailey SR, et al. (2018): When worlds collide: Th17 and Treg cells in cancer and autoimmunity. Cell Mol Immunol 15: 458-469.

50. Zhang X, Koldzic DN, Izikson L, et al. (2004): IL-10 is involved in the suppression of experimental autoimmune encephalomyelitis by CD25+CD4+ regulatory T cells. Int Immunol 16: 249-256.

51. Montero E, Nussbaum G, Kaye JF, et al. (2004): Regulation of experimental autoimmune encephalomyelitis by CD4+, CD25+ and CD8+ T cells: analysis using depleting antibodies. J Autoimmun 23: 1-7.

52. Kohler RE, Caon AC, Willenborg DO, et al. (2003): A role for macrophage inflammatory protein-3 alpha/CC chemokine ligand 20 in immune priming during T cell-mediated inflammation of the central nervous system. J Immunol 170: 6298-6306.

53. Liao F, Rabin RL, Smith CS, et al. (1999): CC-chemokine receptor 6 is expressed on diverse memory subsets of T cells and determines responsiveness to macrophage inflammatory protein 3 alpha. J Immunol 162: 186-194. 
54. Bayry J, Triebel F, Kaveri SV, Tough DF (2007): Human dendritic cells acquire a semimature phenotype and lymph node homing potential through interaction with CD4+CD25+ regulatory T cells. J Immunol 178: 4184-4193.

55. Walker LS (2009): Regulatory T cells overturned: the effectors fight back. Immunology 126: 466-474.

56. Hanig J, Lutz MB (2008): Suppression of mature dendritic cell function by regulatory $\mathrm{T}$ cells in vivo is abrogated by CD40 licensing. J Immunol 180: 1405-1413.

57. Varona R, Zaballos A, Gutierrez J, et al. (1998): Molecular cloning, functional characterization and mRNA expression analysis of the murine chemokine receptor CCR6 and its specific ligand MIP-3alpha. FEBS Lett 440: 188-194.

58. Baba M, Imai T, Nishimura M, et al. (1997): Identification of CCR6, the specific receptor for a novel lymphocyte-directed CC chemokine LARC. J Biol Chem 272: 14893-14898.

59. Greaves DR, Wang W, Dairaghi DJ, et al. (1997): CCR6, a CC chemokine receptor that interacts with macrophage inflammatory protein 3alpha and is highly expressed in human dendritic cells. J Exp Med 186: 837-844.

60. Hromas R, Gray PW, Chantry D, et al. (1997): Cloning and characterization of exodus, a novel beta-chemokine. Blood 89: 3315-3322.

61. Rossi DL, Vicari AP, Franz-Bacon K, et al. (1997): Identification through bioinformatics of two new macrophage proinflammatory human chemokines: MIP-3alpha and MIP-3beta. J Immunol 158: 1033-1036.

62. Liston A, Kohler RE, Townley S, et al. (2009): Inhibition of CCR6 function reduces the severity of experimental autoimmune encephalomyelitis via effects on the priming phase of the immune response. J Immunol 182: 3121-3130. 